\title{
Emergence of a novel recombinant of CV-A5 in HFMD epidemics in Xiangyang, China
}

\author{
Yuting Yu' ${ }^{1}$, Zhiyu Luo ${ }^{1}$, Weiping Jin ${ }^{1}$, Jianyi Mai', Shasha Qian' ${ }^{1}$ Jia Lu', Zhenni Wei ${ }^{1}$, Shengli Meng ${ }^{1}$, \\ Zejun Wang ${ }^{1}$, Xuhua Guan ${ }^{2^{*}}$, Yeqing Tong ${ }^{2^{*}}$ and Shuo Shen ${ }^{1^{*}}$
}

\begin{abstract}
Background: Hand, foot and mouth disease (HFMD) is caused by a variety of enterovirus serotypes and the etiological spectrum worldwide has changed since a large scale of outbreaks occurred in 1997.

Methods: A large number of clinical specimens of HFMD patients were collected in Xiangyang and genotyping was performed by qRT-PCR, conventional PCR amplification and sequencing. Among the 146 CV-A5 detected cases, the complete genome sequences of representative strains were determined for genotyping and for recombination analysis.

Results: It was found that CV-A5 was one of the six major serotypes that caused the epidemic from October 2016 to December 2017. Phylogenetic analyses based on the VP1 sequences showed that these CV-A5 belonged to the genotype D which dominantly circulated in China. Recombination occurred between the CV-A5 and CV-A2 strains with a breakpoint in the 2A region at the nucleotide 3791.

Conclusions: The result may explain the emergence of CV-A5 as one of the major pathogens of HFMD. A multivalent vaccine against HFMD is urgently needed to control the disease and to prevent emerging and spreading of new recombinants.
\end{abstract}

Keywords: Coxsakievirus A5, Recombinantion, Hand, foot and mouse disease

\section{Background}

Hand, foot and mouth disease is caused by a variety of enteroviruses. At least more than 40 enterovirus serotypes were identified in epidemic and etiological surveillances from clinical samples of HFMD patients in mainland China and other countries [1-6]. The major serotypes currently prevailing in China are CV-A6, CV-A10, CV-A16, EV-A71 and other serotypes which either cause sporadic cases or small outbreaks [7]. HFMD is characterized by fever, sore throat, general malaise

\footnotetext{
*Correspondence: Guanxuhua9999@163.com; tongyeqing9999@sina.com; shenshuo1@sinopharm.com

${ }^{1}$ Wuhan Institute of Biological Products, Co. Ltd, Wuhan 430207, People's Republic of China

${ }^{2}$ Xiangyang Center for Disease Control and Prevention, Hubei, People's Republic of China
}

and vesicular eruptions on the hands, feet, oral mucosa and tongue. Severe cases include complications such as encephalitis, meningitis, acute flaccid paralysis, cardiorespiratory failure and even death. Historically, severe and fatal cases are mainly caused by EV-A71; however, in recent years, other serotypes are also responsible for the severity of HFMD cases [6].

Etiological spectrum of HFMD has been changing characteristically driven by the emergence of new predominant enterovirus serotypes such as CV-A6, CV-A10 and other serotypes. The driving forces of evolution are point mutations, intertypic and intratypic recombination [8]. CV-A5 previously caused sporadically HFMD, or herpangina, acute gastroenteritis and onychomadesis at low frequency or small enterovirus outbreaks worldwide [9-20]. Indeed, CV-A5 was recently associated 
with a relatively large proportion of HFMD cases in a largescale etiological investigation in Xiangyang performed in our laboratory. A total of 3, 703 case HFMD samples were collected and the typeable case number was 3201, which were genotyped by blasting and comparison with sequences of enteroviruses in GenBank. Of the typeable 3201 specimens, $\mathrm{CV}-\mathrm{A} 6$ was the predominating serotype followed by CV-A16, CV-A10, CV-A5, CV-A2 and EV-A71 and the ratios were $51.47 \%$ (1906/3201), 15.3\% (490/3201), $11.56 \%$ (370/3201), 4.56\% (146/3201), 3.78\% $(121 / 3201)$ and $3.03 \%(97 / 3201)$, respectively. It seemed that these six serotypes were popular at the same time in Xiangyang [21].

CV-A5 is a member of species A in the genus Enterovirus within the family Picornaviridae in the order Picornavirales [22]. It has been further divided into clades $\mathrm{A}$, $\mathrm{B}, \mathrm{C}$ and D based on the divergence of the VP1 nucleotide sequence [23]. The single-stranded, polyadenylated, positive-sense RNA genome is proximately 7400 nucleotides in length. The genome contains an open reading frame (ORF) flanked by the untranslated regions (UTR) at both $5^{\prime}$ - and $3^{\prime}$-terminals with a peptide VPg encoded by viral $3 \mathrm{~B}$ protein, which covalently linked to the $5^{\prime}$ UTR. The polyprotein is co-translationally cleaved by viral proteases 2 Apro, 3Cpro (or 3CDpro) into precursors such as P1, P2, P3, VP0, 3CD and mature structural proteins VP1-VP4 (1A-1D) and nonstructural proteins 2A-2C, 3A-3D.

In this study, the complete genome sequences of three $\mathrm{RD}$ cell isolates of $\mathrm{CV}$-A5 were determined. These three isolates were the representative strains of $146 \mathrm{CV}-\mathrm{A} 5$ clinical specimens in an epidemic of HFMD outbreak in Xiangyang in 2017. Phylogenetic analysis revealed that a novel recombinant emerged carrying the $5^{\prime}$-terminal sequence of CV-A5 and the $3^{\prime}$-terminal sequence of $\mathrm{CV}-\mathrm{A} 2$ with the recombination junction located at $2 \mathrm{~A}$ region. The recombination may be responsible for the high proportion of CV-A5 HFMD cases among all enteroviruses detected. This novel recombinant may explain the outbreak caused by CV-A5 and emphasize the role played by recombination in the emerging, prevailing and changing of etiological spectrum of HFMD-associated enteroviruses.

\section{Materials and methods Collection of clinical data}

Rectal swabs were collected from children with the symptoms of HFMD from three hospitals in Xiangyang, Hubei, China. The samples were transferred by viral transport medium Youkang (Beijing, China) containing Hanks balanced salt solution. The specimens were stored at $-80{ }^{\circ} \mathrm{C}$. The viral RNAs were detected by real time RT-PCR and conventional PCR. All specimens were
Table 1 Universal primers for Enterovirus VP1

\begin{tabular}{lll}
\hline Name & Primer sequence $^{\text {a }}\left(\mathbf{5}^{\prime}-\mathbf{3}^{\prime}\right)$ & Position $^{\mathbf{b}}$ (nt) \\
\hline 292 & 5'-MIGCIGYIGARACNGG-3' $^{\prime}$ & $2612-2627$ \\
222 & 5'-CICCIGGIGGIAYRWACAT-3' $^{\prime}$ & $2969-2951$ \\
\hline
\end{tabular}

a IUB fuzzy code; I: deoxyinosine; $M: A / C ; Y, C / T ; R: A / G ; N: A / T / G / C ; W: A / T$

${ }^{\mathrm{b}}$ The location relative to the genome of the poliovirus type I Mahoney strain

inoculated in monolayer of RD and Vero cells for virus isolation.

\section{Enterovirus isolation and RNA extraction}

All of CV-A5 strains were sampled from April to July in 2017, among them CV-A5 R3474, CV-A5 R3487, CV-A5 R3490 were collected in April and isolated in RD cells and Vero cells by conventional methods [24], respectively. Samples were identified by molecular typing method. The viral RNAs were extracted directly from cell lysates.

\section{VP1 region sequencing and typing of $\mathrm{CV}-\mathrm{A} 5$ strain}

The VP1 regions were amplified by enterovirus VP1 universal primers (292 and 222) [25] listed in Table 1. The reaction was performed by adding $2 \mu \mathrm{l}$ reaction buffer, $0.5 \mu \mathrm{l}$ cDNA template, $3.7 \mu \mathrm{l}$ enzyme mixture, $0.6 \mu \mathrm{l}$ forward primer, $0.6 \mu \mathrm{l}$ reverse primer $(1.0 \mathrm{ng} / \mu \mathrm{l}$ each), and nuclease-free water to be a total volume of $20 \mu \mathrm{l}$. The PCR amplification was performed under the following conditions: $94{ }^{\circ} \mathrm{C} 5 \mathrm{~min} ; 35$ cycles of $94{ }^{\circ} \mathrm{C} 30 \mathrm{~s}, 55^{\circ} \mathrm{C} 30 \mathrm{~s}$, and $72{ }^{\circ} \mathrm{C} 30 \mathrm{~s} ; 72{ }^{\circ} \mathrm{C} 10 \mathrm{~min}$. VP1 sequences were compared with reference sequences available in GenBank. Virus strains which showed more than $75 \%$ nucleotide similarity with known enterovirus serotypes were considered to be the same serotype.

\section{Full-length genome sequencing}

The complete genome of $\mathrm{CV}$-A5 strains were sequenced for further genetic characterization. PCR conditions:

Table 2 Primers used for PCR amplification and sequencing of CV-A5 genome

\begin{tabular}{lll}
\hline Name & Primer sequence $\left(\mathbf{5}^{\prime} \mathbf{- 3}^{\prime} \mathbf{)}\right.$ & Position $(\mathbf{n t})$ \\
\hline A5 1F & TTAAAACAGCCTGTGGGTTGTACC & $1-24$ \\
A5 6F & GCAATCCACTATGGTAGGACAACT & $1998-2021$ \\
A5 9F & GCACCTTTTCAGTGAGATTCGTT & $3128-3250$ \\
A5 9R & ACCTACTGCAAGCATGAGATG & $3630-3610$ \\
A5 13R & AACCCAGCAAAGAGCTTGTAGATA & $4825-4806$ \\
A5 20F & AAGAGAGTCTACGCCCTGGAG & $4366-4386$ \\
A5 23R & TTGACCCCTTGTTCATCCACTAAT & $5588-5565$ \\
A5 24F & TCACTGCTGAGAAGGAATATCAGG & $5407-5430$ \\
A5 24R & CCAAAATGTATCTGGATTGCACCC & $6585-6562$ \\
A5 polyA & AGTCAAGTTACATAGTAGGCTACAGTA & $7370-7419$ \\
& ACTGCTTTTTTTTTTTTTTTT & \\
\hline
\end{tabular}




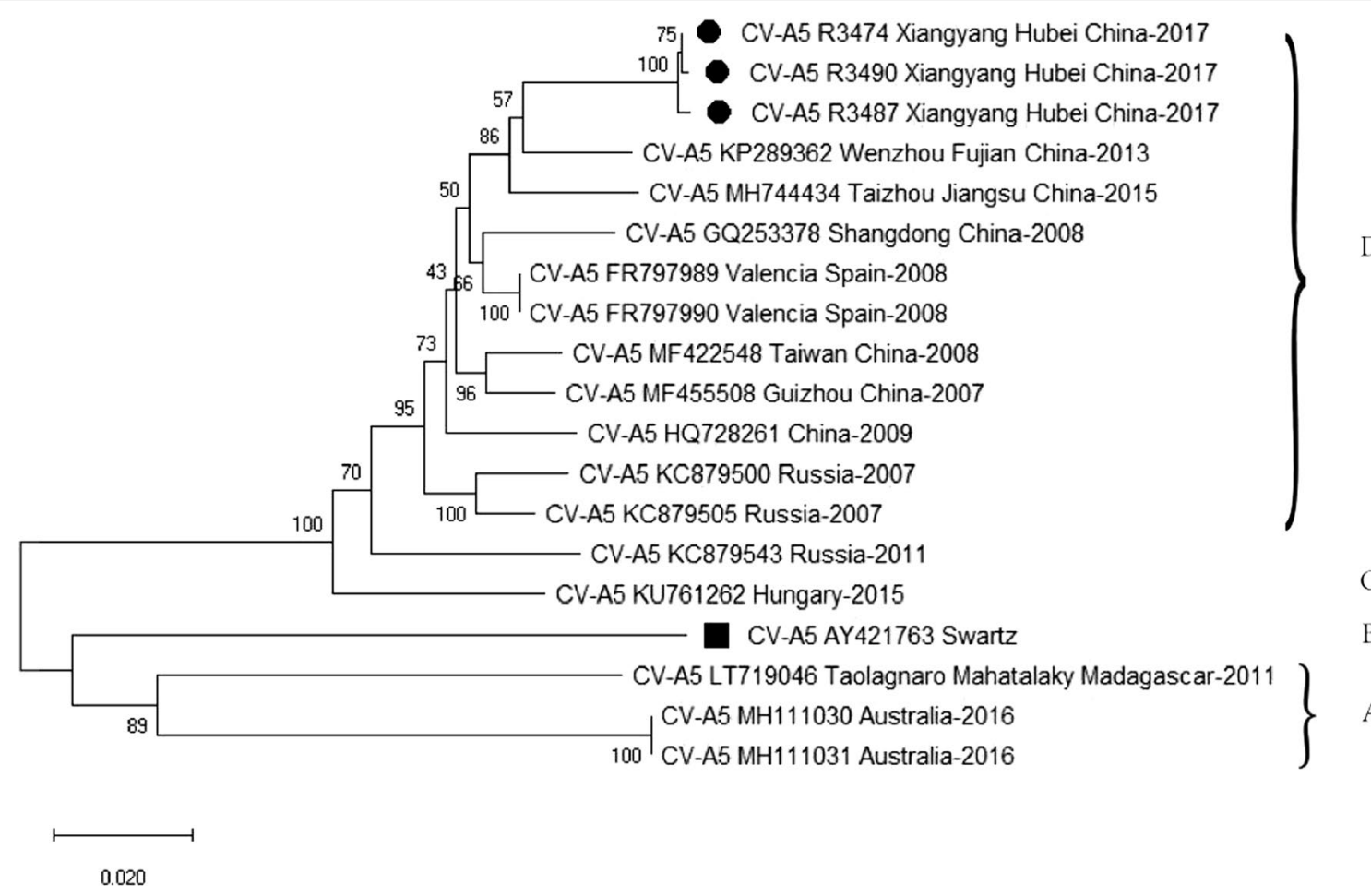

D

Fig. 1 Phylogenetic analysis of Xiangyang CV-A5 isolates and reference strains from Genbank based on the VP1 sequences. Filled circle represented the strains isolated from Xiangyang and Filled square labeled the CV-A5 prototype Swartz. The GenBank accession no, isolation year and place of each reference strain were indicated. The phylogenetic tree was reconstructed by the neighbor-joining method on the basis of the Kimura (2-parameter) model implemented in MEGA-X. Bootstrap values of 500 was used for evaluation. The genotype demarcation was shown

$94{ }^{\circ} \mathrm{C} 5$ min; $94{ }^{\circ} \mathrm{C} 30$ s, $52{ }^{\circ} \mathrm{C} 30$ s, $72{ }^{\circ} \mathrm{C} \mathrm{kb} / 30$ s, 35 cycles; $72{ }^{\circ} \mathrm{C} 10 \mathrm{~min}$. Primers used were listed in Table 2.

\section{Phylogenetic analysis and recombination analysis}

The nucleotide homology among three CV-A5 strains from Xiangyang was 99.6\%. The CV-A5 R3487 strain was selected to compare its nucleotide and amino acid sequence with those of other enterovirus by Megalign software. The phylogenetic tree was generated by MEGA$\mathrm{X}$ software and the evolutionary distance was calculated with the neighbor-joining method using 500 boostraps for evaluation. The recombination was analyzed by using the MEGA-X and SimPolt software, through the Bootstaps method (500 Boostraps Replications) and the Kimura (2-parameter) model mapping, respectively. The Recombination Detection Program (RDP) was used for the detection of potential recombinant sequences and localization of possible recombination breakpoints.

\section{Nucleotide sequence accession number}

16 CV-A5 strains VP1 nucleotide sequence downloaded from Genebank (888 nucleotides) and the full-length genome sequences of CV-A5 R3474, CV-A5 R3487,
CV-A5 R3490 strains were determined in this study. The complete genome sequence of CV-A5 R3487, CV-A5 R3474, CV-A5 R3490 were deposited in GeneBank under the accession no MN663160, OK334537, OK334538.

\section{Results}

Detection and cell isolation of CV-A5

Among 4415 cases of clinically diagnosed HFMD, 3781 (85.64\%) were confirmed by real time RT-PCR and conventional PCR to be accounted for enteroviruses from Oct 2016 to Dec 2017 at Xiangyang. These included 78 cases of EV-A71 (1.77\%) and 3703 cases of (83.87\%) nonEV-A71 enteroviruses. Of these 3703 samples, serotyping and genotyping were performed by sequencing of amplified PCR fragments. The results showed that $86.44 \%$ of HEVs (3201/3703) were typeable by blasting and comparison with sequences of enteroviruses in GenBank. Among them, 146 cases were CV-A5 (4.56\%). The result indicated that $\mathrm{CV}$-A5 emerged as one of the six main serotypes associated with HFMD. All samples were subjected to cell isolation in RD and Vero cells. Among them, 188 strains of enteroviruses belonging to 18 serotypes were isolated in either RD or Vero cells. Four strains were 
Table 3 The sequence differences between CV-A5 R3474, CV-A5 R3487 and CV-A5 R3490

\begin{tabular}{|c|c|c|c|c|c|c|c|}
\hline & \multirow[t]{2}{*}{ Position (Nt/Aa) } & \multicolumn{2}{|c|}{ CV-A5-R3474 } & \multicolumn{2}{|c|}{ CV-A5-R3487 } & \multicolumn{2}{|c|}{ CV-A5-R3490 } \\
\hline & & $\mathrm{Nt}$ & $\mathrm{Aa}$ & $\mathrm{Nt}$ & $\mathrm{Aa}$ & $\mathrm{Nt}$ & $\mathrm{Aa}$ \\
\hline VP4 & $876 / 43$ & G & $\mathrm{R}$ & A & $\mathrm{R}$ & A & $\mathrm{R}$ \\
\hline \multirow[t]{2}{*}{ VP2 } & $1115 / 54$ & A & $\mathrm{T}$ & A & $\mathrm{T}$ & G & $A$ \\
\hline & $1377 / 141$ & $C$ & $P$ & A & $P$ & C & $P$ \\
\hline \multirow[t]{3}{*}{ VP3 } & $1818 / 33$ & G & $E$ & A & $E$ & A & $E$ \\
\hline & 2095/125 & $\mathrm{T}$ & A & $\mathrm{T}$ & A & C & A \\
\hline & $2356 / 212$ & $\mathrm{~T}$ & I & C & I & C & 1 \\
\hline \multirow[t]{4}{*}{ VP1 } & 2809/123 & A & $E$ & A & E & G & $E$ \\
\hline & $2872 / 144$ & $\mathrm{~T}$ & $\mathrm{~N}$ & A & K & T & $\mathrm{N}$ \\
\hline & $2885 / 149$ & C & $P$ & $\mathrm{~T}$ & L & C & $P$ \\
\hline & $3302 / 288$ & C & S & $\mathrm{T}$ & P & C & S \\
\hline \multirow[t]{2}{*}{$2 \mathrm{~A}$} & $3420 / 31$ & G & L & A & L & G & $L$ \\
\hline & $3670 / 114$ & $\mathrm{~T}$ & $L$ & $\mathrm{~T}$ & L & C & $L$ \\
\hline \multirow[t]{2}{*}{$2 C$} & $4261 / 62$ & C & $\mathrm{N}$ & C & $\mathrm{N}$ & $\mathrm{T}$ & $\mathrm{N}$ \\
\hline & $4917 / 281$ & $\mathrm{~T}$ & C & C & C & C & C \\
\hline \multirow[t]{2}{*}{$3 \mathrm{~A}$} & $5070 / 3$ & C & $P$ & $\mathrm{~T}$ & $P$ & C & $P$ \\
\hline & $5205 / 48$ & C & P & $\mathrm{T}$ & $P$ & C & $P$ \\
\hline \multirow[t]{3}{*}{$3 C$} & $5397 / 4$ & $\mathrm{~T}$ & $L$ & C & $L$ & C & $L$ \\
\hline & $5774 / 129$ & A & G & A & G & G & G \\
\hline & $5868 / 161$ & C & $\mathrm{H}$ & $\mathrm{T}$ & $\mathrm{H}$ & C & $\mathrm{H}$ \\
\hline \multirow[t]{6}{*}{$3 \mathrm{D}$} & $5983 / 17$ & G & V & A & I & A & 1 \\
\hline & $6174 / 80$ & $\mathrm{~T}$ & $\mathrm{H}$ & C & $\mathrm{H}$ & C & $\mathrm{H}$ \\
\hline & $6769 / 278$ & T & $\mathrm{N}$ & $\mathrm{T}$ & $\mathrm{N}$ & C & $\mathrm{N}$ \\
\hline & $6874 / 313$ & G & $\mathrm{T}$ & G & $\mathrm{T}$ & A & $\mathrm{T}$ \\
\hline & $7076 / 381$ & C & S & C & S & $\mathrm{T}$ & $S$ \\
\hline & $7215 / 427$ & A & K & G & K & A & K \\
\hline
\end{tabular}

isolated in RD cells and the isolation rate of CV-A5 in RD cells was low (4/146 of CV-A5 positive swabs). Three of four isolates grew at titers higher than $1 \times 10^{7} \mathrm{CCID}_{50} / \mathrm{ml}$ and were also adapted to Vero cells. The complete nucleotide sequences of Vero-adapted isolates were determined for recombination analysis.

\section{Determination of the complete genome}

The complete genome of the three $\mathrm{CV}$-A5 isolates were determined and the homology of nucleotide between each strain were higher than $99.5 \%$ by blasting analysis (Fig. 1). The detailed sequence differences between these three CV-A5 strains were showed in Table 3. The genome of the representative strain CV-A5 R3487/XY/CHN/2017 (abbreviated as CV-A5 R3487) is 7356 nucleotides in length. The size of ORF is 6576 bases encoding a polyprotein of 2191 amino acids, which is flanked by the $5^{\prime}$ and $3^{\prime}$ UTR (747 and 81 nucleotides, respectively). The genome is polyadenylated at the terminus of the $3^{\prime}$ UTR. The $5^{\prime}$-terminal of primers (18 nucleotides) designed in this experiment were based on the sequence of CV-A5 prototype Swartz.

\section{Serotyping and phylogenetic analysis of Xiangyang isolates}

Blasting analysis based on the VP1 gene showed that homologies of the CV-A5 strains in Xiangyang with the prototype Swartz were $81.6 \%-82.0 \%$ for nucleotide and 94.9\%-95.9\% for amino acid, respectively. In contrast, homologies with other serotypes were below $75 \%$ for nucleotide and $85 \%$ for amino acid, respectively. Based on the criterion of the molecular typing recommended by Oberste [9], Xiangyang isolates were identified as $\mathrm{CV}-\mathrm{A} 5$ serotype. To determine the phylogenetic relationship between Xiangyang CV-A5 strains and other CV-A5 strains available in the Genbank, a phylogenetic tree was constructed based on the complete VP1 sequence with 16 CV-A5 strains representing genotypes A-D, including six reported Chinese strains (Fig. 1). The phylogenetic dendrogram indicated that the three Xiangyang CV-A5 strains clustered into genotype D and shared identities of $95.9-96.3 \%$ and $98.3 \%-99.3 \%$ in nucleotide and amino acid sequences, respectively. Moreover, the Xiangyang isolates displayed the highest similarity with Wenzhou CV-A5 isolate in 2013 [26]. However, there was a clear 

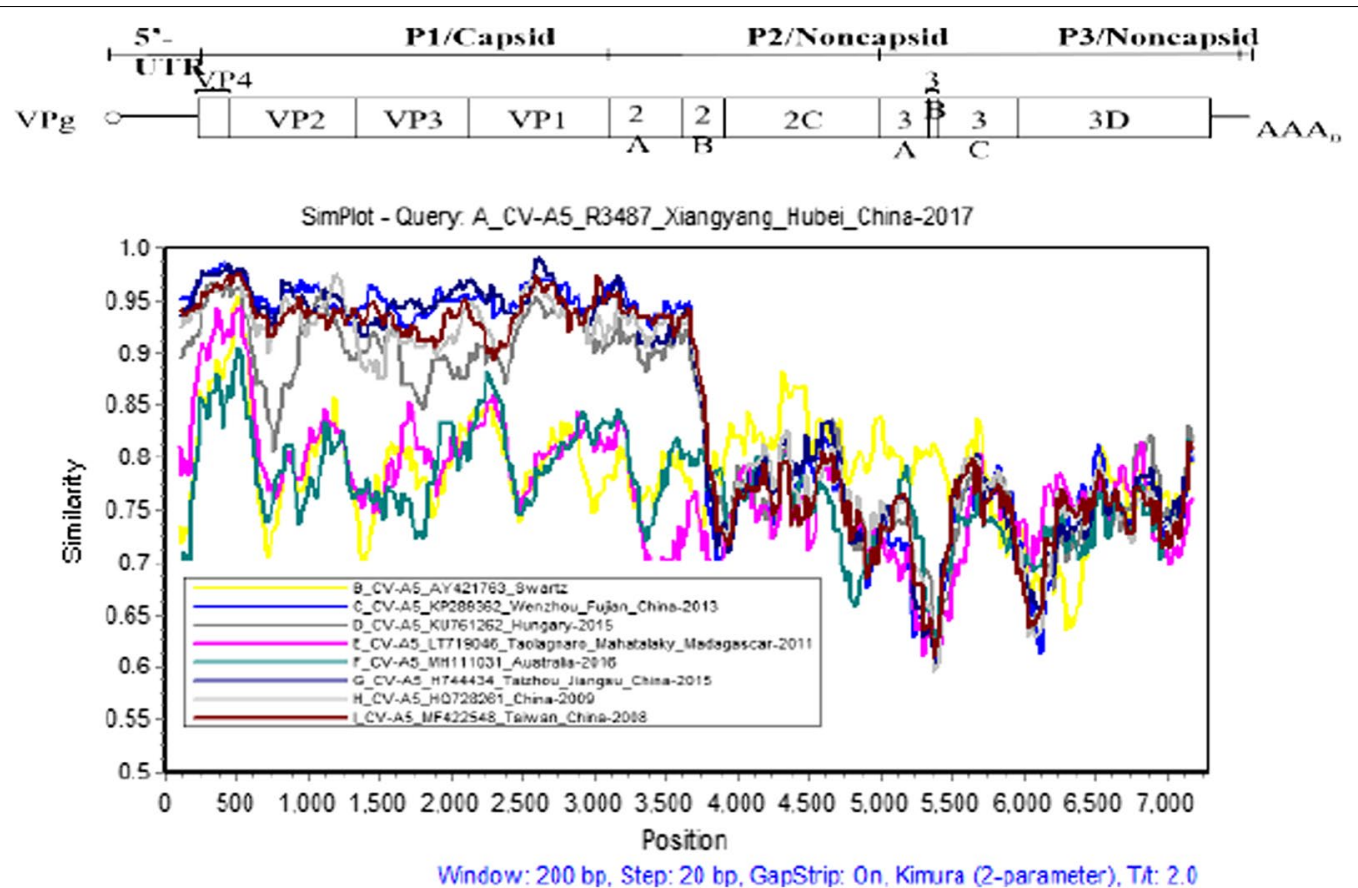

Fig. 2 Similarity plot analysis of the whole genome of the Xiangyang CV-A5 R3487 strain. The similarity was calculated in a sliding window size of 200 nucleotides (nt) moving in 20 nt steps, using the Kimura 2-parameter distance method. The sequence of CV-A5 R3487 strain served as a query sequence

genetic distinction between the Xiangyang $\mathrm{CV}$-A5 isolates and the prototypes Swartz or other reported CV-A5 isolates elsewhere.

\section{Recombination analysis}

Previously, we reported that a Xiangyang CV-A2 R1580 strain was a recombinant which shared high sequence identity with CV-A5 prototype Swartz in P2 region [27]. To investigate the genetic mechanism of the emerging CV-A5 associated with HFMD epidemic at Xiangyang in 2017, the viral genome regions of CV-A5 R3487 virus, including the $5^{\prime}$ UTR, P1, P2 and P3 regions, were phylogenetically analyzed for genetic relationships with other eight CV-A5 reference sequences.

The multi-sequences were scanned by SimPlot software (Version 3.5.1) to generate the similarity plot showed in Fig. 2. Compared with other eight CV-A5 strains, the similarities of CV-A5 R3487 decreased dramatically from the $2 \mathrm{~B}$ to $3^{\prime}$ UTR region. This result implied that there might be a recombination event with other serotypes in the $2 \mathrm{~A} / 2 \mathrm{~B}$ junction of CV-A5 R3487 strain. As shown in Fig. $3 \mathrm{a}$, in the 2B, 2C, P3 and $3^{\prime}$ UTR regions, CV-A5 R3487 displayed the highest similarity with Shenzhen CV-A2 isolate (KX595284) in 2015 and CV-A2 R1580 strain isolated in Xiangyang in 2017. However, in the $5^{\prime}$ UTR, P1 and 2A regions, the sequence similarities were relatively higher with Wenzhou CV-A5 strain (KP289362) isolated in 2013. Bootscanning analysis was performed, which confirmed a recombination event occurred at the nucleotide (nt) position 3791 in the $2 \mathrm{~A}$ region of strain CV-A5 R3487 (Fig. 3b). Taken together, these results suggested that a recombinant event may occur between CV-A5 R3487 and CV-A2 originated from an imported (Wenzhou) or a local (Xiangyang) CV-A2 strain.

Finally, up- and down-stream regions before and after the breakpoint 3791 were aligned for sequence comparison. The result showed that the $5^{\prime}$-half (nt 1-3791) and $3^{\prime}$-half (nt 3792-7356) were clustered with CV-A5 and CV-A2 strains tested, respectively (Fig. 4a, b). The novel CV-A5 R3487 recombinant obtained the 5' UTR, P1 and partial 2A regions and downstream partial 2A, 2B, 2C, P3 and $3^{\prime}$ UTR regions of CV-A2 KX595284 strain. A novel $\mathrm{CV}-\mathrm{A} 5 / \mathrm{CV}-\mathrm{A} 2$ recombinant emerged and caused an outbreak of HFMD in Xiangyang in 2017.

\section{Discussion}

RNA viruses exist as quasispecies when they evolve in nature and are passaged in cells or experiment animals [28-30]. The lack of fidelity and proofreading of the RNA-dependent RNA polymerase (RdRp) of these viruses results in high error rates through nucleotide misincorporation during genome replication, which could explain how these viruses rapidly mutate and evolve [31, 
Fig. 3a

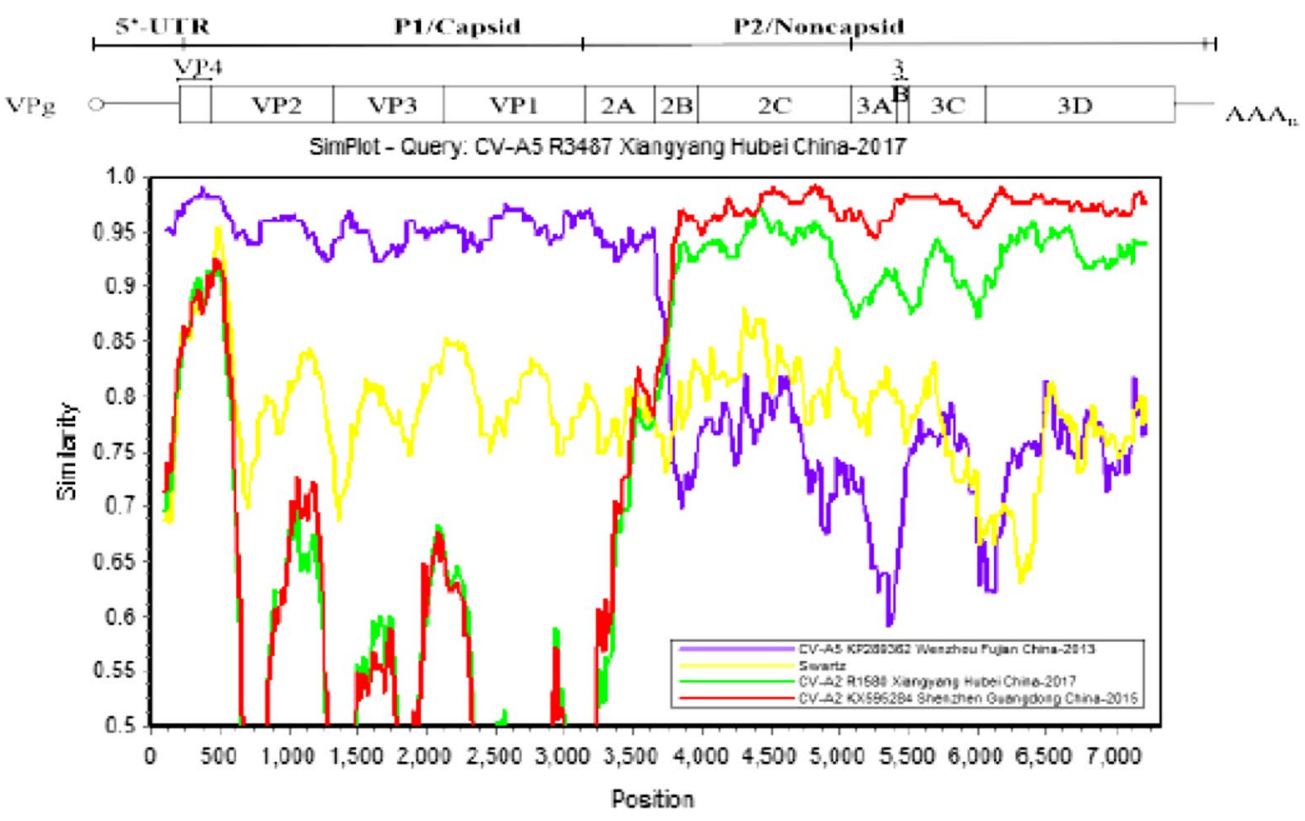

Fig. 3b

\begin{tabular}{|c|c|c|c|c|c|c|c|c|c|c|}
\hline \multicolumn{3}{|c|}{ 5'-UTR } & \multicolumn{2}{|c|}{ P1/Capsid } & \multicolumn{5}{|c|}{ P2/Noncapsid } & \multirow[t]{2}{*}{ P3/Noncapsid } \\
\hline & $v p$ & & & & & & & & & \\
\hline$V P g$ & $\circ$ & VP2 & VP3 & VP1 & $2 A$ & $2 B$ & $2 \mathrm{C}$ & $3 \mathrm{AP}$ & $3 C$ & $3 D$ \\
\hline
\end{tabular}

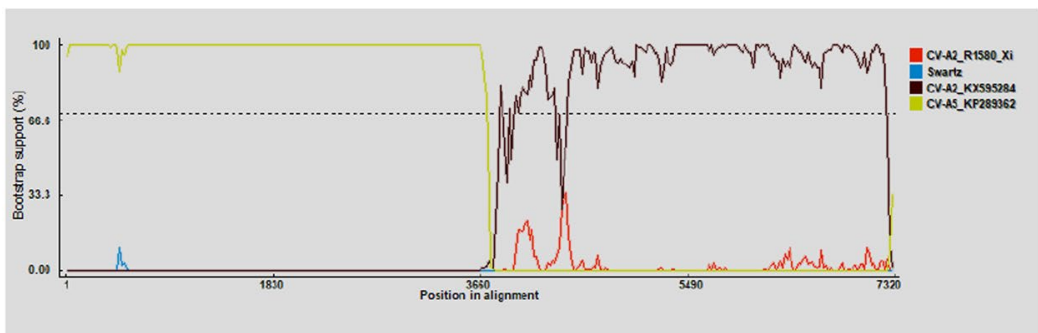

Fig. 3 Identification of recombination of Xiangyang CV-A5 R3487 based on the whole genome. Similarity analysis (a) and bootscaning analysis (b) were performed in a $200 \mathrm{nt}$ sliding window. Each point indicated similarity between the Xiangyang CV-A5 R3487 strain and other EV-A strains in a $20 \mathrm{nt}$ moving step. Kimura (2-parameter) model was used in the analysis. The strains indicated by orange, green, blue and grey were Xiangyang CV-A2 R1580, Shenzhen CV-A2 (KX595284), CV-A5 prototype Swartz and Wenzhou CV-A5 (KP289362) isolates, respectively. The sequence of CV-A5 R3487 strain served as a query sequence

32]. The emergence, evolution and virulence/epidemics of RNA viruses including enteroviruses are also driven by recombination. The mechanisms involve template switching $[33,34]$ by the RdRp via an intermediate step of duplicated segments [35] or the replication-independent joining of RNA molecules [36]. It is believed that recombination can correct deleterious mutations and provide advantage for emerging new viruses [37].

Numerous intertypic and intratypic recombination events have been reported mostly in the same enterovirus species within and between serotypes of species A to D. Co-circulation and co-infection of several enteroviruses facilitate the emergence of recombinants. Recombination may act against Muller's Ratchet [38,39] and as a counterbalancing force against high mutation rates [37] by eradicating deleterious mutations. It may also lead to the combination of advantageous properties from various genomes into a new one such as emergence of drug resistance or even evasion from the immune system. 


\section{Fig. 4a}

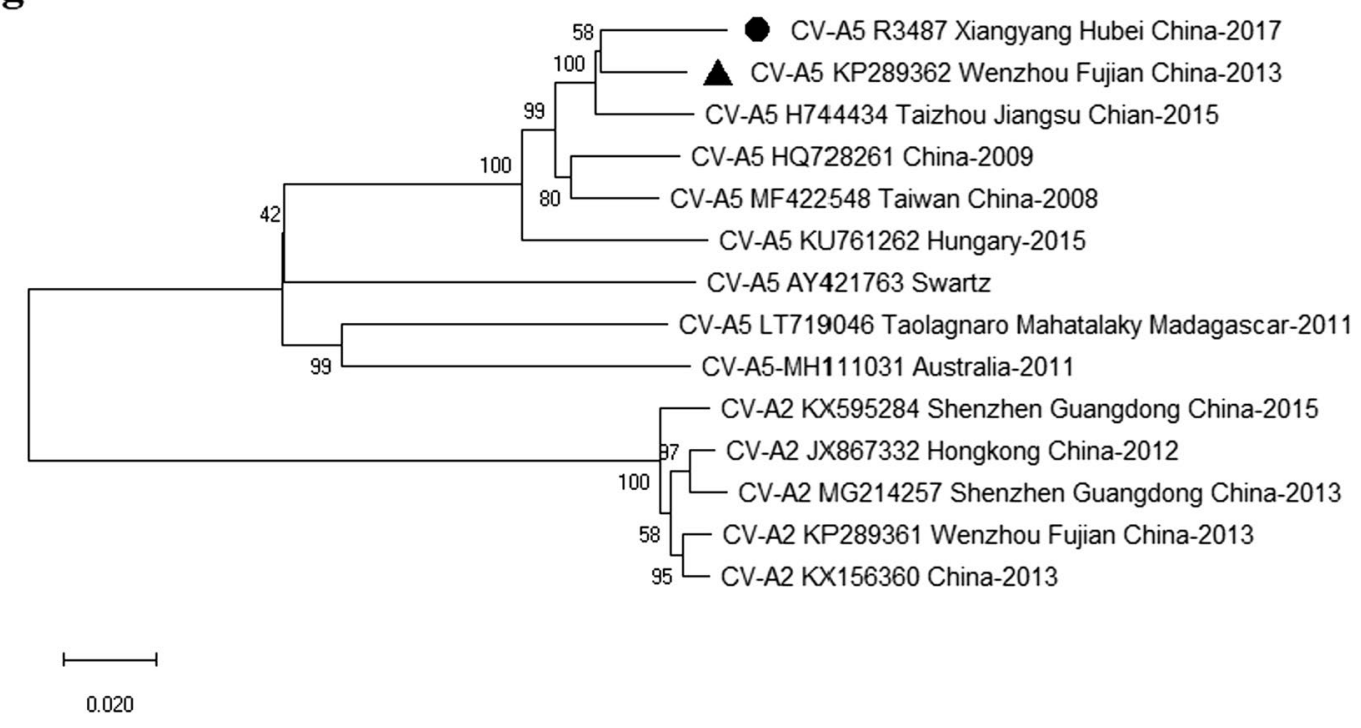

Fig. 4b

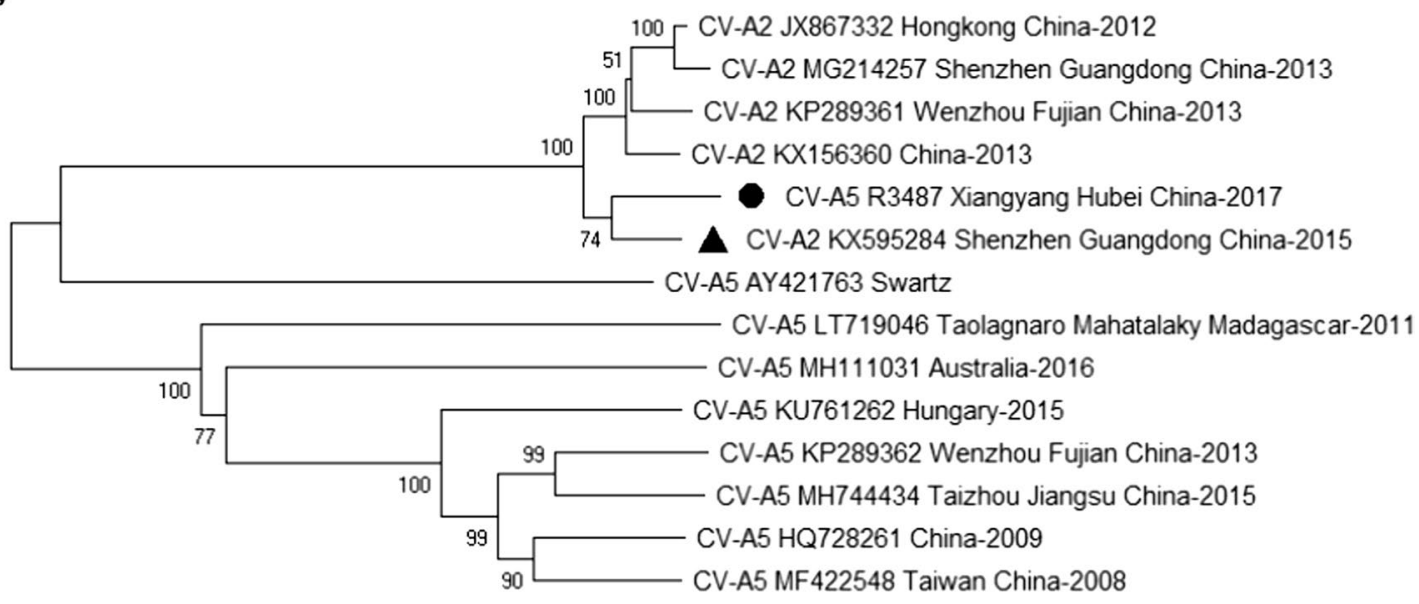

0.020

Fig. 4 Phylogenetic trees of genetic fragments for Xiangyang CV-A5 R3487 and other CV-A5 and CV-A2 strains, including 5' UTR-2A (nt 1-3791) (a) and 2A-3' UTR (nt 3792-7404) (b) region, were illustrated in figure. Isolate of Xiangyang CV-A5 R3487 was marked with filled circle, the strain of Wenzhou CV-A5 (KP289362) or the strain of Shenzhen CV-A2 (KX595284) was marked with filled triangle in figure a or b, respectively. The phylogenetic trees were reconstructed using neighbor-joining method with the Kimura (2-parameter) model. Bootstrap values of 500 was used for evaluation

Emergence of new recombinants is often responsible for the infection, transmission, pathogenesis of these viruses and the consequence may be deadly sometimes. For example, a recombinant CV-A2 caused the deaths of several children in Hong Kong in 2012 [40] and a recombinant of CV-B4 resulted in a fatal case of HFMD in Guangxi in 2012 [41]. Emergence and spread of novel recombinants are often associated with an outbreak of a disease in human population [42]. It is believed that a recombinant of EV-A71 with CV-A16 was one of the reasons behind the outbreak of HFMD epidemic of in Fuyang, China in 2008 [43].

The prevailing EV-A71 is a replication domains donor to other species A enteroviruses, as recently reported research on recombination [44]. CV-A5 also dominated its replication part of the genome to EV-A17 and the 
recombinant circulated in Eastern Asia [45]. There is evidence that suggests recombination contribute to the switch of dominant serotypes and changes of clinical manifestation [46]. In the outbreak of HFMD in Shanghai in 2012, a recombinant of CV-A6 emerged, which resulted in more generalized skin lesions than normal symptoms induced with non-recombinant CV-A6 [46]. Between 2012 and 2013 in many cities and areas, CV-A6 and CV-A10 have emerged and even became the dominant serotypes [4]. Changes of etiological spectrum may result from mutations and recombination. Following the largescale vaccination of EV-A71 vaccine in China, it is important to closely monitor the new emerging pathogens for disease control measures and for development of multivalent vaccines against HFMD [47].

In Xiangyang, China in 2017, the HFMD outbreak was mainly caused by six main serotypes including CV-A6, A16, A10, A2, A5 and EV-A71. Meanwhile, other 12 serotypes were also detected in clinical samples of HFMD patients at very low proportions. The co-circulation of multiple serotypes increased the chances of co-infection and intertypic recombination [44]. In a period of nine months in 2017, CV-A2 and CV-A5 were co-circulating with case numbers of 117 and 146, respectively. Complete sequence analysis of 3 representative CV-A5 isolates showed that they were recombinants between $\mathrm{CV}$-A5 with a breakpoint at $2 \mathrm{~A}$ region.

In this study, 8 pairs of primers spanning the recombination hot spots based on published paper [8] were designed for sequence amplification. Unfortunately, by agarose gel electrophoresis, only 7 fragments of 5 samples could be amplified in the right size of PCR products. Interestingly, through blast comparison, we found that 2 of them had the possibility of recombination, CV-A5 R1382 and CV-A5 R1307. The similarity in 2A-2C region between CV-A5 R1382 and CV-A4 (MH086049.1) was 97.95\%, and the similarity in 3A-3C region between CV-A5 R1307 and CV-A2 (MN419014.1) was 98.74\%. CV-A5 R1382 and CV-A5 R1307, like CV-A5 R3474, CV-A5 R3487, CV-A5 R3490, were also collected in April, 2017. The deficiency of this experiment was that it was difficult to obtain the complete genome of CV-A5 R1382 and CV-A5 R1307. Although one recombination hot spot was determined, further studies should be performed to clarify the potential recombination.

The limitation of this study was that only 8 and 4 cell isolates were obtained for $\mathrm{CV}-\mathrm{A} 2$ and $\mathrm{CV}-\mathrm{A} 5$, respectively. The complete genome sequences of all CV-A5 samples were difficult to complete due to degradation of viral RNA. The ratio of parental strains to recombinants was not known. It was not clear whether recombination occurred in or spread to Xiangyang, as the parental donor strain providing P2 and P3 region was not detected.
Nonetheless, further study should be performed to investigate the origin, evolution of CV-A5 parental strain and recombinant, and its pathogenicity in mouse model and human. Following the largescale vaccination of EV-A71 vaccine in China, it is worthwhile to closely monitor the new emerging pathogens for disease control and for development of multivalent vaccines against HFMD.

\section{Conclusions}

This study presents complete sequence analysis of 3 representative $\mathrm{CV}$-A5 isolates showed that they were recombinants between CV-A5. This result may explain the emergence of CV-A5 as one of the major pathogens of HFMD epidemics in Xiangyang, China.

\section{Abbreviations}

HFMD: Hand, foot and mouse disease; ORF: Open reading frame; RDP: Recombination detection program; RdRp: RNA dependent RNA polymerase.

\section{Acknowledgements}

We thank all the patients for their cooperation. We thank all the proof-readers and editors for their contribution.

\section{Authors' contributions}

YTY and SS wrote the main manuscript text, analyzed the date and prepared figures and tables. YTY and SS designed the work. YTY, ZYL, WPJ, JYM, SSQ and ZNW collected and analyzed the data. SS, SSQ, JL, XHG, YQT, SLM, and ZJW critically revised the manuscript for important intellectual content. All authors read and approved the final manuscript.

\section{Funding}

This work was supported by the Ministry of Science and Technology of the People's Republic of China (2015ZX09102021). The funding body played no role in the design of the study and collection, analysis, and interpretation of data and in writing the manuscript.

\section{Availability of data and materials}

All of the sequence data have been deposited. The datasets generated and/ or analysed during the current study are available in the NCBI. The GenBank number of the sequence involved in the manuscript are: MN663160(CV-A5 R3487), OK334537(CV-A5 R374), OK334538(CV-A5 R3490), OK393668(CV-A5 R1307), OK393669(CV-A5 R1382). The datasets used and analyzed during the current study are available from the corresponding author on reasonable request.

\section{Declarations}

\section{Ethics approval and consent to participate}

This work was approved by Medical Ethics Committee of Xiangyang Disease Control Center. Verbal informed consents were obtained from legal guardians of all patients prior to the collection of rectal swab samples. Verbal informed consent was the preferred option for this study since it is more efficient for patient guardians. Every patient got detailed information of the study, and all of them had the ability to comprehend the study and was able to reason and make one's own decision. All of the verbal informed consent of the patients were documented in a form. Medical Ethics Committee of Xiangyang Disease Control Center also approved the procedure for verbal consent.

\section{Consent for publication}

Not applicable.

Competing interests

The authors declare that they have no competing interests. 
Received: 19 January 2021 Accepted: 21 October 2021

Published online: 24 November 2021

\section{References}

1. Wei SH, Huang YP, Liu MC, et al. An outbreak of coxsackievirus A6 hand, foot, and mouth disease associated with onychomadesis in Taiwan, 2010. BMC Infect Dis. 2011:11:346.

2. Fujimoto T, lizuka S, Enomoto M, et al. Hand, foot, and mouth disease caused by coxsackievirus A6, Japan, 2011. Emerg Infect Dis. 2012;18(2):337-9.

3. Di B, Zhang Y, Xie HP, et al. Circulation of coxsackievirus A6 in hand-footmouth disease in Guangzhou, 2010-2012. Virol J. 2014;11:157.

4. Guan HY, Wang J, Wang CR, et al. Etiology of multiple non-EV71 and nonCVA16 enteroviruses associated with hand, foot and mouth disease in Jinan, China, 2009-June 2013. PLoS ONE. 2015;10(11):e0142733.

5. Yao X, Bian LL, Lu WW, et al. Enterovirus spectrum from the active surveillance of hand foot and mouth disease patients under the clinical trial of inactivated enterovirus A71 vaccine in Jiangsu, China, 2012-2013. J Med Virol. 2015;87(12):2009-17.

6. Ji H, Fan H, Lu PX, et al. Surveillance for severe hand, foot, and mouth disease from 2009 to 2015 in Jiangsu province: epidemiology, etiology, and disease burden. BMC Infect Dis. 2019:19(1):79.

7. $\mathrm{Xu} \mathrm{MH,} \mathrm{Su} \mathrm{LY,} \mathrm{Cao} \mathrm{LF,} \mathrm{et} \mathrm{al.} \mathrm{Genotypes} \mathrm{of} \mathrm{the} \mathrm{enterovirus} \mathrm{causing} \mathrm{hand}$ foot and mouth disease in Shanghai, China, 2012-2013. PLoS ONE. 2015;10(9):e0138514

8. Nikolaidis M, Mimouli K, Kyriakopoulou Z, et al. Large-scale genomic analysis reveals recurrent patterns of intertypic recombination in human enteroviruses. Virology. 2019;526:72-80

9. Zhu B, Zhong JY, Xia HM, et al. Etiology of hand, foot and mouth disease in Guangzhou in 2008. Chin J Pediatr. 2010;48(2):127-30.

10. Davia JL, Bel PH, Ninet VZ, et al. Onychomadesis outbreak in Valencia, Spain associated with hand, foot, and mouth disease caused by enteroviruses. Pediatr Dermatol. 2011;28(1):1-5.

11. Wang ZG, Liu XL, Yang TT, et al. Etiology of hand, foot and mouth disease in Qingdao during 2008-2009. Chin JVirol. 2011;27(5):438-41.

12. Ding $S, L i C R$, Wang $Y H$, et al. Epidemiological characteristics of hand-footmouth disease and gene features of coxsackievirus A6 and A5 in Xiaogan area from 2014-2016. Occup Health. 2018;34(8):1038-42.

13. Pham NTK, Thongprachum A, Trinh QD, et al. Detection and genetic characterization of enterovirus strains circulating among children with acute gastroenteritis in Japan during 2014-2016. Infect Genet Evol. 2018;61:16-9.

14. Huang WC, Huang LM, Lu CY, et al. Atypical hand-foot-mouth disease in children: a hospital-based prospective cohort study. Virol J. 2013;10:209.

15. Yamashita $\mathrm{T}$, Ito $\mathrm{M}$, Taniguchi $\mathrm{A}$, et al. Prevalence of coxsackievirus a5, a6, and a10 in patients with herpangina in Aichi prefecture, 2005. Jpn J Infect Dis. 2005:58(6):390-1.

16. Baek KA, Yeo SG, Lee BH, et al. Epidemics of enterovirus infection in Chungnam Korea, 2008 and 2009. Virol J. 2011;8:297.

17. Park SH, Choi SS, Oh SA, et al. Detection and characterization of enterovirus associated with herpangina and hand, foot and mouth disease in Seoul, Korea. Clin Lab. 2011:57(11-12):959-67.

18. Park KS, Lee $B H$, Baek KA, et al. Enteroviruses isolated from herpangina and hand-foot-and-mouth disease in Korea children. Virol J. 2012;9:205.

19. Puenpa J, Mauleekoonphairoj J, Linsuwanon P, et al. Prevalence and characterization of enterovirus infections among pediatric patients with hand foot mouth disease, herpangina and influenza like illness in Thailand, 2012. PLoS ONE. 2014;9(6):198.

20. Xu SJ, Ren Y, Hao XQ, et al. Characteristics of the pathogen for hand-footand-mouth disease in Longhua district, Shenzhen in 2018. China Trop Med. 2019;19(3):267-70

21. Meng XD, Tong YQ, Wei ZN, et al. Epidemical and etiological study on hand, foot and mouth disease following EV-A71 vaccination in Xiangyang, China. Sci Rep. 2020;10(1):20909.

22. Zell R, Delwart E, Gorbalenya AE, et al. ICTV virus taxonomy profile: Picornaviridae. J Gen Virol. 2017;98(10):2421-2.

23. Oberste MS, Maher K, Kilpatrick DR, et al. Molecular evolution of the human enteroviruses: correlation of serotype with VP1 sequence and application to picornavirus classification. J Virol. 1999;73(3):1941-8.

24. Zhang Y, Yan DM, Zhu SL, et al. Type 2 vaccine-derived poliovirus from patients with acute flaccid paralysis in China: current immunization strategy effectively prevented its sustained transmission. J Infect Dis. 2010;202(12):1780-8.

25. Oberste MS, Nix WA, Maher K, et al. Improved molecular identification of enteroviruses by RT-PCR and amplicon sequencing. J Clin Virol. 2003:26(3):375-7.

26. Guo WP, Lin XD, Chen YP, et al. Fourteen types of co-circulating recombinant enterovirus were associated with hand, foot, and mouth disease in children from Wenzhou, China. J Clin Virol. 2015;70:29-38.

27. Luo ZY, Wang ZJ, Meng SL, et al. Analysis of mutation and recombination for coxsackievirus A2 isolated in patients with hand, foot and mouth disease in 2017 in Xiangyang city. Prog Microbiol Immunol. 2019;4:19-21.

28. Domingo E, Sabo D, Taniguchi T, et al. Nucleotide sequence heterogeneity of an RNA phage population. Cell. 1978;13(4):735-44.

29. Domingo E, Holland JJ. RNA virus mutations and fitness for survival. Annu Rev Microbiol. 1997;51:151-78.

30. Fang SG, Shen S, Tay FPL, et al. Selection of and recombination between minor variants lead to the adaptation of an avian coronavirus to primate cells. Biochem Biophys Res Commun. 2005:336(2):417-23.

31. Lei X, Cui S, Zhao Z, et al. Etiology, pathogenesis, antivirals and vaccines of hand, foot, and mouth disease. Natl Sci Rev. 2015:2:268-84.

32. Woodman A, Arnold JJ, Cameron CE, et al. Biochemical and genetic analysis of the role of the viral polymerase in enterovirus recombination. Nucleic Acids Res. 2016;44(14):6883-95.

33. Arnold JJ, Cameron CE. Poliovirus RNA-dependent RNA polymerase (3Dpol) is sufficient for template switching in vitro. J Biol Chem. 1999;274(5):2706-16.

34. Kirkegaard K, Baltimore D. The mechanism of RNA recombination in poliovirus. Cell. 1986;47(3):433-43.

35. Lowry K, Woodman A, Cook J, et al. Recombination in enteroviruses is a biphasic replicative process involving the generation of greater-than genome length "imprecise" intermediates. PLoS Pathog. 2014;10(6):e1004191.

36. Gmyl AP, Belousov EV, Maslova SV, et al. Nonreplicative RNA recombination in poliovirus. J Virol. 1999;73(11):8958-65.

37. Xiao YH, Rouzine IM, Bianco $S$, et al. RNA recombination enhances adaptability and is required for virus spread and virulence. Cell Host Microbe. 2017;22(3):420.

38. Felsenstein J. The evolutionary advantage of recombination. Genetics. 1974;78(2):737-56.

39. Muller $\mathrm{HJ}$. The relation of recombination to mutational advance. Mutat Res. 1964;106:2-9.

40. Yip CCY, Lau SKP, Woo PCY, et al. Recombinant coxsackievirus A2 and deaths of children, Hong Kong, 2012. Emerg Infect Dis. 2013;19(8):1285-8.

41. Hu YF, Du J, Zhao R, et al. Complete genome sequence of a recombinant coxsackievirus B4 from a patient with a fatal case of hand, foot, and mouth disease in Guangxi, China. JVirol. 2012;86(19):10901-2.

42. Liu YJ, Zhang FF, Fu C, et al. Combination of intratypic and intertypic recombinant events in EV71: a novel evidence for the "triple-recombinant"strains of genotype A viruses in mainland China from 2008 to 2010. Virus Genes. 2015;50(3):365-74.

43. Zhang Y, Zhu Z, Yang WZ, et al. An emerging recombinant human enterovirus 71 responsible for the 2008 outbreak of hand foot and mouth disease in Fuyang city of China. Virol J. 2010;7:94.

44. Lee LM, Gong YN, Hsieh TH, et al. Discovery of enterovirus A71-like nonstructural genomes in recent circulating viruses of the enterovirus A species. Emerg Microbes Infect. 2018;7(1):111.

45. Shimizu H, Utama A, Yoshii K, et al. Enterovirus 71 from fatal and nonfatal cases of hand, foot and mouth disease epidemics in Malaysia, Japan and Taiwan in 1997-1998. Jpn J Infect Dis. 1999;52(1):12-5.

46. Feng XB, Guan WC, Guo YF, et al. A novel recombinant lineage's contribution to the outbreak of coxsackievirus A6-associated hand, foot and mouth disease in Shanghai, China, 2012-2013. Sci Rep. 2015;5:11700.

47. Jin WP, Lu J, Zhang XY, et al. Efficacy of coxsackievirus a5 vaccine candidates in an actively immunized mouse model. JVirol. 2021;95(6):e01743-e1820.

\section{Publisher's Note}

Springer Nature remains neutral with regard to jurisdictional claims in published maps and institutional affiliations. 